\title{
Solid-state Structure and Formation of Organized Molecular Films for Comb Copolymer Containing N-vinylcarbazole
}

\author{
Hiroko Hoshizawa, Ryosuke Masuya, Toru Masuko, and Atsuhiro Fujimori* \\ Department of Polymer Science and Engineering, Faculty of Engineering, Yamagata University, Yonazawa 992-8510, Japan \\ Fax: +81-238-26-3073, e-mail: bp0311@dipfr.dip.yz.yamagata-1.ac.jp
}

We investigated the molecular orientation of organized molecular films with regard to solid-state structures for newly synthesized comb copolymers with $\mathrm{N}$-vinylcarbazole (NVCz) by X-ray diffraction (XRD), surface pressure-area $(\pi-A)$ isotherm and in-plane XRD. In the bulk state, hydrogenated and fluorinated comb copolymers formed side-chain crystals for a two-dimensional lattice spacing of 4.2 and $5.0 \AA$, respectively. In this case, it was suggested that the carbazole rings in the main-chain were arranged in opposition to each other. From the results of the DSC measurements, sharp-shaped melting peaks appeared in the relatively lower temperature side of the thermograms. This result supported the formation of side-chain crystals in the synthesized comb copolymers. These monolayers of the copolymers on the water surface were extremely condensed, except for the fluorocarbon: $\mathrm{NVCz}=1: 1$ copolymer. From the in-plane XRD measurements of multilayers on solids, the changes in the two-dimensional lattice structure of fluorinated comb copolymer films containing NVCz units, as opposed to their bulk state, were confirmed. It seems that these structural changes are caused by the stronger $\pi-\pi$ interaction between the carbazole rings rather than the van der Waals interaction between fluorocarbons.

Key words: N-vinylcarbazole, Comb copolymer, Side-chain crystal, Organized molecular films, Two-dimensional lattice

\section{INTRODUCTION}

In recent times, many researchers have attempted to utilize poly-N-vinylcarbazole (PNVCz) as organic light emitting diodes, organic transistors, and host compounds for organic electro luminescence ${ }^{1,2}$ because it is well known that $\mathrm{PNVC}_{2}$ exhibits a property identical to that of organic semiconductors. ${ }^{3}$ Further, it is expected that PNVCz can be used to realize a hologram memory material because of the high refractive index (greater than 1.68) of its monomer. ${ }^{4}$

On the other hand, it is essential to control the molecular orientation and the arrangement at the monomolecular level in order to drive the functionality of these organic molecular devices in an efficient manner. However, in many cases, it is difficult to control the molecular arrangement and the homopolymer packing because PNVCz itself easily forms an amorphous polymer. $^{3}$

Previously, we have investigated the control of solid-state structures and the formation of organized films for the newly synthesized comb copolymers containing hydrogenated and fluorinated side-chains. ${ }^{6}$ These comb polymers form a side-chain crystal in the bulk state by van der Waals interaction between the side chains and the extremely stable condensed monolayers at the air/water interface. If the incorporation of bulky and flat functional groups in the main chain of side-chain crystalline polymers is realized, it may be possible to control the arrangement of bulky groups at the molecular level, regardless of the low crystallinity of the polymers.

In this study, the fine structure in the solid-state of the synthesized comb polymers containing $\mathrm{N}$-vinylcarbazole (NVCz) in the main chain obtained by co polymerization with hydrogenated or fluorinated long-chain compounds was investigated by wide-angle $X$-ray diffraction. Further, the molecular arrangement of the organized molecular films in comb copolymers containing $\mathrm{NVCz}$ units were estimated by in-plane $\mathrm{X}$-ray diffraction.

\section{EXPERIMENTAL}

\subsection{Materials}

The comb copolymers used in this study were obtained by the copolymerization of $\mathrm{NVCz}$ with octadecyl acrylate (OA) or 2-(perfluorodecyl)ethyl acrylate $\left(\mathrm{FF}_{10} \mathrm{EA}\right)$ at several monomer ratios. Copolymerizations were carried out in a solution of acetone at $50^{\circ} \mathrm{C}$ for $48 \mathrm{~h}$ using $1 \mathrm{~mol} \%$ azobisisobutyronitrile (AIBN) as an initiator. These monomers and the initiator were purchased from Tokyo Kasei Co. Ltd. and Daikin Fine Chemicals Co. Ltd., and were used without further purification. The precipitated polymers were washed with acetone until they were free of monomers. The syndiotactic poly-FF ${ }_{10} \mathrm{EA}$ homopolymer was obtained from a $1 \mathrm{M}$ rad ${ }^{60} \mathrm{Co} \gamma$-ray irradiated post-polymerization according to the procedure described in the previous work. ${ }^{7}$ The $\gamma$-ray irradiation was carried out at the Japan Atomic Energy Institute at Takasaki. The comb copolymer compositions were determined by ${ }^{1} \mathrm{H}$ 
NMR (Nihon Densi Co. Ltd. EX270 NMR) spectroscopy. The tacticity of fluorinated homopolymer obtained by ${ }^{1} \mathrm{H}$ NMR analysis according to the reference was found to be almost syndiotactic (Diad: $58 \%$ ). The molecular weights of several copolymers with higher ratios of OA units were estimated to be approximately $\left.\overline{\mathrm{Mw}}=4.65 \times 10^{4} \overline{(\mathrm{Mw}} / \overline{\mathrm{Mn}} \approx 1.96\right)$ on the basis of GPC (JASCO-860-CO) measurements. The molecular weights of fluorinated homopolymers and copolymers with higher ratios of $F_{10} E A$ units were estimated to be above thousand. These values were estimated from the intrinsic viscosity $[\eta]$, which is equal to $0.12 \sim 0.54$, for these trifluoroacetic acid solutions at $30{ }^{\circ} \mathrm{C}$ by using the relation $[\eta]=\mathrm{KM}^{\mathrm{a}}$, where $\mathrm{K}=$ $0.24 \sim 0.25 \times 10^{-4}$ and $\alpha=0.75 \sim 0.78$. The values of $K$ and $\alpha$ were assumed from the results of the viscosity-averaged molecular weight of poly-alkyl acrylate. ${ }^{8}$

According to the theory of the Q-e scheme proposed by T. Alfry and C.C. Price, ${ }^{9}$ these comb polymers form alternating copolymers. In this case, the e values of $\mathrm{NVCz}$ and $\mathrm{OA}$ are -1.40 and +1.12 , respectively. Thus, it appears that the NVCz:long-chain acrylate copolymers almost form ideal alternating copolymers, especially for the $1: 1$ monomer ratio.

\subsection{Structural estimation of bulk copolymers.}

The packing modes of several copolymers in the crystalline phase were examined by $X$-ray powder diffraction measurements using a Rigaku Rad-rA diffractometer, which was equipped with a graphite monochrometer, with $\mathrm{CuKa}$ radiation at $40 \mathrm{kV}$ and $200 \mathrm{~mA}$.

2.3 Formation of copolymer monolayers on water surface and estimation of molecular arrangement in the films.

The monolayers of the comb copolymer with $\mathrm{NVCz}$ were spread from the chloroform or chloroform/trifluoroacetic acid $=90 / 10(\mathrm{v} / \mathrm{v})$ mixed solutions (about $10^{-4} \mathrm{M}$ ) onto the distilled water (about $18 \mathrm{M} \Omega \cdot \mathrm{cm}$ ). The surface pressure-area $(\pi-A)$ isotherms of the polymers were measured on a film balance (Kyowa Kaimen Kagaku Co. Ltd., compression speed: 5 $\AA^{2} /$ repeating units $\cdot \min$ ) at $15{ }^{\circ} \mathrm{C}$. These fluorinated comb copolymers formed extremely condensed monolayers. These monolayers were transferred to the solid substrates at $15^{\circ} \mathrm{C}$ and at a proper surface pressure $\left(15 \sim 25 \mathrm{mNm}^{-1}\right)$ by the Langmuir-Blodgett (LB), and horizontal lifting method (HLM) to obtain the alternating Y-type film and the nonalternating $X-$ film, respectively.

The in-plane spacing of the two-dimensional lattice of the films was determined by analysis using an X-ray diffractometer for different geometrical arrangements ${ }^{10}$, 11 (Bruker AXS, MXP-BX, CuKa radiation, $40 \mathrm{kV}, 40 \mathrm{~mA}$, an instrument specially made to order) equipped with a parabolic graded multilayer mirror. The monomolecular level resolution of this in-plane
XRD apparatus was realized by applying the $\mathrm{X}$-rays at an incident angle of $0.2^{\circ}$ and by slow scanning at $0.05^{\circ} / 20 \mathrm{~s}$.

\section{RESULTS AND DISCUSSION}

3.I Estimation of fine structures for comb copolymers in bulk state.

Figure 1 shows the WAXD profiles of the comb copolymers containing $\mathrm{NVCz}$ with several side-chain ratios. From these profiles, interesting systematic changes were confirmed in the short-spacing region, which depended on the side-chain ratios. Further, it is concluded that PNVCz forms an amorphous polymer because of the appearance of two types of halos and the absence of a peak.

In addition, from the profiles of poly-OA and poly-FF ${ }_{10} \mathrm{EA}$, short spacing peaks based on the sub-cell formation of side-chains were confirmed at 4.2 and $5.0 \AA$, respectively. According to Platés review, these peaks correspond to (100) reflection. $^{12}$ In the small angle region, long spacing peaks were clearly observed in the profile of poly-FF ${ }_{10} \mathrm{EA}$ at approximately $20.3 \AA$, whereas no peaks were observed in the low angle side in the profile of poly-OA. Poly-FF ${ }_{10} \mathrm{EA}$ forms a highly ordered layer structure along the direction of the c-axis. In accordance with the previous work, it appears that these long spacing peaks are defined at the $(002)$ reflection ${ }^{13}$ because poly-FF ${ }_{10} \mathrm{EA}$ forms a double layer structure and the calculated length of the fluorocarbon side-chain is examined at approximately $20 \AA$. Based on computer simulation estimations, the $d$-spacing from the (001) reflection peak of Poly-OA was expected to be approximately $50 \AA$ (double layer spacing), although poly-OA did not form the layer structure. And further, in Fig. 1(a), the intensity of the $(100)$ reflection $\left(d_{100}=4.2 \AA\right)$ gradually decreased with an increase in the $O A$ ratio. However, in the case of the NVCz:FF ${ }_{10} \mathrm{EA}$ system shown in Fig. 1(b), the (100) reflection $\left(d_{100}=5.0 \AA\right)$ suddenly disappeared between the ratios 1:3 and 1:5. The reason for this phenomenon might be the formation of any block unit of fluorocarbons in the NVCz:FF ${ }_{10} \mathrm{EA}=1: 3$ copolymer.

In the case of the NVCz:OA $=1: 1$ copolymer, a long spacing peak appeared at 32.7 $\AA$. This value is larger than the simulated value
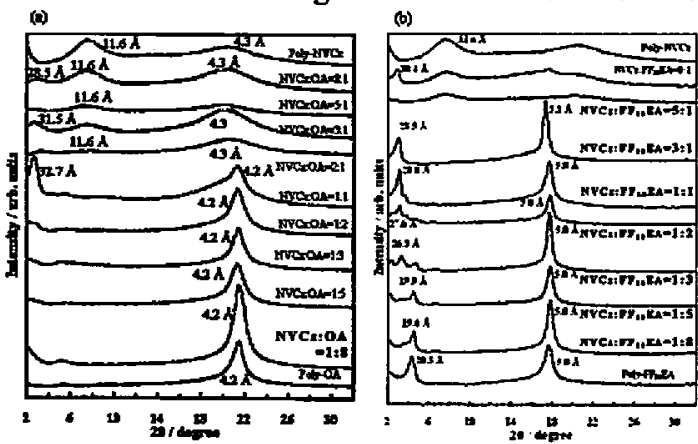

Figure 1 XRD profiles of comb copolymers containing NVCz with several ratios. (a)OA:NVCz, (b)FF 10 EA:NVCz copolymers. 
of the molecular period (approximately $25 \AA$ ) of poly-OA. In addition, the long spacing of the NVCz:FF ${ }_{10} E A=1: 1$ copolymer shifted to the lower angle side at approximately $8 \AA$ as compared to that of Poly-FF ${ }_{10} E A$. From the results of simple simulation for molecular dynamics, it is found that molecular length of long-chain acrylates combined with $\mathrm{NVCz}$ are longer at $7.7 \AA$ than amphiphiles uncombined with carbazole groups. Further, short spacing peaks were also observed in the profiles of both kinds of copolymers at the same position as that in the corresponding profiles of each comb-like homopolymer. These experimental findings also indicate the formation of side-chain crystalline polymers containing $\mathrm{NVCz}$ units, although the NVCz:OA $=1: 1$ copolymer includes amorphous regions.

These XRD profiles indicate the possibility of the structural control of bulky functional groups related to the formation of side-chain crystals and their crystallinity reflected on the peak width. These results contain very important and clear information on the formation of side-chain crystalline polymers with two-dimensional sub-cell structures.

\subsection{Molecular arrangement of organized molecular films for comb copolymers.}

On the basis of these experimental results, we attempt to utilize the comb copolymers containing $\mathrm{NVCz}$ units as the material for the formation of monolayers on the water surface. Figure 2(a) and (b) show the surface pressure-area $(\pi-A)$ isotherms for the monolayers of the $\mathrm{NVCz}: \mathrm{OA}$ and $\mathrm{NVCz}_{\mathrm{FF}} \mathrm{FEA}_{10}$ copolymers, respectively, on distilled water at $15^{\circ} \mathrm{C}$. It has been observed that these comb polymer monolayers stabilize considerably and form condensed films. However, the limiting molecular areas extrapolated to $\pi$ $=0$ from the steep region of the $\pi-A$ curves of the NVCz:OA and NVCz:FF ${ }_{10} E A=5: 1,2: 1$ polymers were

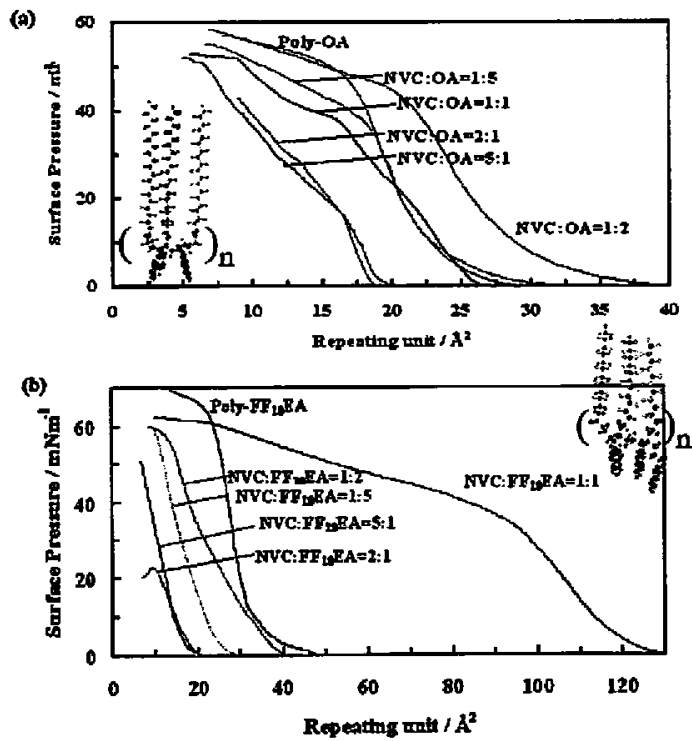

Figure $2 \pi-A$ isotherms of monolayers on the water surface of (a) $\mathrm{NVCz}: \mathrm{OA}$ copolymers and (b) $\mathrm{NVCz}: \mathrm{FF}_{10} \mathrm{EA}$ copolymers at $15^{\circ} \mathrm{C}$. below $20 \AA^{2}$ repeating unit of hydrophobic parts, which is an extremely small value when compared to the general hydrogenated amphiphiles. It is possible that these films on the water surface were no longer "monolayers." In other words, at the moment when the surface pressure was detected, there was a possibility that a piled-up film (in other words termed "collapsed monolayer") was formed on the water surface and the surface pressure increased with compression. This speculation will be supported by the following atomic force microscopic observation of the monolayers on a solid. These experimental findings are caused by lack of hydrophilicity. That is to say, carbazole groups are not hydrophilic groups but hydrophobic groups. The formation of a homogeneous monolayer of these comb copolymers with a high $\mathrm{NVCz}$ content is difficult.

The other copolymer monolayers exhibited a proper limiting area, while the large molecular area of the NVCz:FF ${ }_{10} \mathrm{EA}=1: 1$ monolayer in the isotherm attracted our attention. This material is an almost ideal alternating coploymer, and the van der Waals interaction between fluorocarbons is generally weak. It appears that the orientation of the carbazole rings is almost flat with respect to the water surface because fluoroside chains cannot be packed together completely.

Figure 3 shows the in-plane X-ray diffraction profiles of several transferred films (20 layers) of comb copolymers containing $\mathrm{NVCz}$. These profiles indicate the packing mode in the organized molecular films of the copolymer systems. In the case of the NVCz:OA copolymer multilayers in Fig. 3(a), which were transferred by the LB and HLM methods, all two-dimensional lattices form a hexagonal packing at a lattice spacing of $4.2 \AA$. These values are almost identical to the powder XRD profiles in the bulk state. Therefore, these data indicate that the packing mode of the side-chain crystal of a two-dimensional film is nearly the same as that of the three-dimensional crystal.

Here, very interesting results are shown in Fig. 3(b). Organized molecular films of almost all NVCz:FF ${ }_{10} E A$ copolymers do not form a similar packing in their bulk state. In the case of a copolymer with high $\mathrm{FF}_{10} \mathrm{EA}$ ratios, hexagonal packing was formed at a lattice

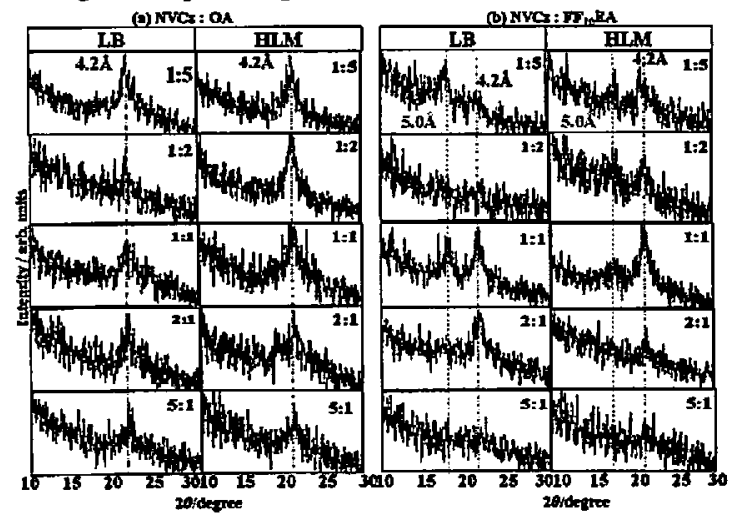

Figure 3 In-plane XRD profiles of multilayers of comb copolymers containing NVCz (20 layers). 
spacing of $5.0 \AA$. However, the in-plane XRD profile of the $\mathrm{NVCz}_{\mathrm{FF}} \mathrm{FEA}_{10}=1: 1$ copolymer films, which form expanded monolayers on the water surface, exhibits two kinds of short spacing peaks at 4.2 and $5.0 \AA$. From this result, it is inferred that a distorted orthorhombic packing of a side-chain lattice is formed. Further, in the case of high $\mathrm{NVCz}$ ratios, an isotropic hexagonal packing for a two-dimensional lattice spacing of $4.2 \AA$ is constructed although the value of $4.2 \AA$ is generally too narrow to pack fluorocarbons in lattice.

In order to interpret these phenomena, we describe the schematic models in Fig. 4 . It is assumed that the structural changes in fluoro-copolymer are caused due to the competition between the forces formed in a twoor three-dimensional crystal, namely, the "weak van der Waals interaction between fluorocarbons" and the "relatively strong $\pi-\pi$ interaction between carbazole rings." In the case of the monolayer formation on the water surface, the van der Waals force is not sufficient to pack the hydrophobic chains, especially those of the 1:1 copolymer. Therefore, these polymer molecules are rearranged by the $\pi-\pi$ interaction between the carbazole rings at the moment when a transfer to the solid substrate occurs. Consequently, it appears that the side-chain packing changes with the rearrangement of the carbazole groups. Probably, the most suitable spacing between the carbazole groups for the $\pi-\pi$ interaction to work is $4.2 \AA$, which is identical to the value of the spacing between the hydrogenated side-chain crystals.

As mentioned previously, it is possible to control the orientation of the functionalised

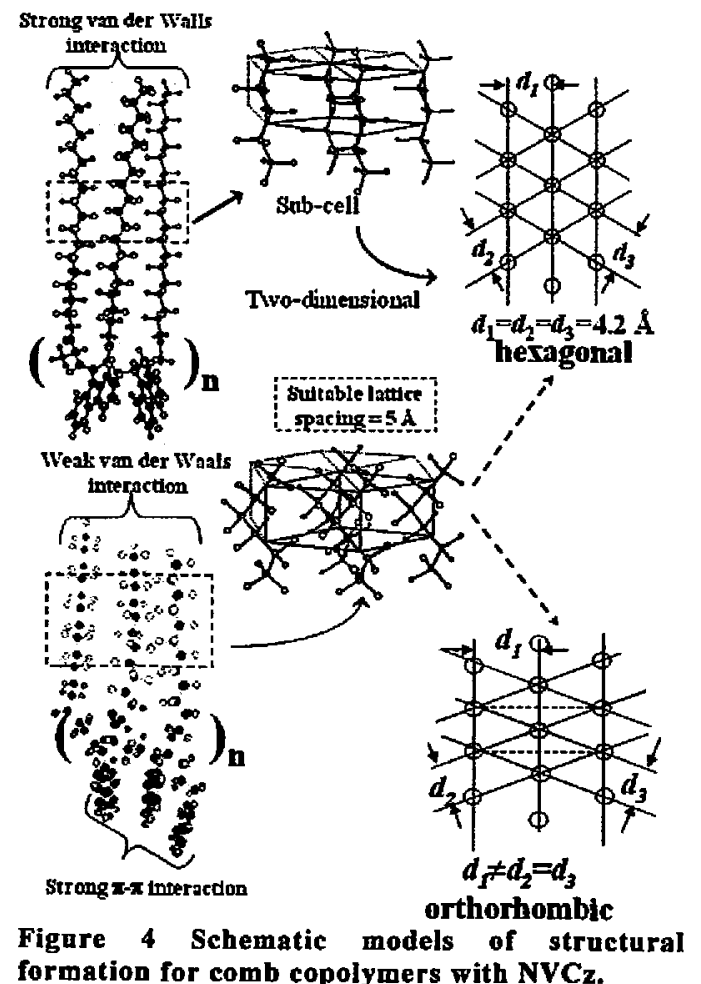

carbazole group arranged by copolymerization with long-chain vinyl compounds (resultant comb copolymer) and the method of organized molecular films.

\section{CONCLUSION}

We investigated the molecular arrangement of mono and multilayers with regard to the three-dimensional crystal structure of newly synthesized comb copolymers with the carbazole group by XRD, $\pi-A$ isotherm and in-plane XRD. In the solid state, hydrogenated and fluorinated comb copolymers containing $\mathrm{NVCz}$ form side-chain crystals at a lattice spacing of 4.2 and $5.0 \AA$, respectively. It was suggested that the carbazole rings incorporated in the copolymer main chain were arranged in opposition to each other. The Langmuir monolayers on the water surface of these copolymers formed extremely condensed monolayers, except for the fluorocarbon: $\mathrm{NVCz}=1: 1$ copolymer. From the in-plane XRD analysis of multilayers in solids, the changes in the two-dimensional lattice structure of fluorinated comb copolymers containing $\mathrm{NVCz}$ units, as opposed to their bulk state, were confirmed. It seems that these structural changes are caused by the stronger $\pi-\pi$ interaction between the carbazole rings rather than the van der Waals interaction between the fluorocarbon side-chains.

\section{REFERENCES}

[1] H. Hoegl, J. Phys. Chem., G9, 255, (1965).

[2] J. M. Pearson, M. Stolka, "Poly(N-vinylcarbazole)" in Polymer monograph, vol. 6, New York: Gordon and Breach, (1981).

[3] K. R. Choudhury, M. Samoc, A. Patra, P. N. Prasad, J. Phys. Chem. B, 108(5), 1556, (2004).

[4] M. T. R. Laguna, J. Gallego, F. Mendicuti, E. Saiz, M.P. Tarazona, Macromolecules, 35(20), 7782, (2006).

[5] C. Maertens, P. Dubois, R. Jerome, P.A. Blanche, P.C. Lemaire, J. Polym. Sci., Part B, Polym. Phys., 38(1), 205, (2000).

[6] R. Masuya, N. Ninomiya, A. Fujimori., H. Nakahara, T. Masuko, J. Polym. Sci., Part B, Polym. Phys., 44, 416, (2006).

[7] A. Fujimori, H. Saitoh, and Y. Shibasaki, $J$. Thermal. Anal., 57, 631, (1999).

[8] J. G. Fee, W. S. Port, L. P. Witnauer, J. Polym Sci., 33, 95, (1958).

[9] T. Alfrey, C. C. Price, J. Polym. Sci., 2, 101, (1947).

[10] A. Fujimori, Y. Sugita, H. Nakahara, E. Ito, M. Hara, N. Matsuie, K. Kanai, Y. Ouchi, K. Seki, Chem. Phys. Lett, 387, 345, (2004).

[11] A. Fujimori, T. Araki, H. Nakahara, E. Ito, M. Hara, H. Ishii, Y. Ouchi, K. Seki, Chem. Phys. Lett., 349, 6, (2001).

[12] N. A. Platé, V. P. Shibaev, J. Palym. Sci., Macromol. Rev., 8, 117, (1974).

(Received December 10, 2006;Accepted February 8, 2007) 